\title{
Management of chronic anterior uveitis relapses: efficacy of oral phospholipidic curcumin treatment. Long-term follow-up
}

This article was published in the following Dove Press journal:

Clinical Ophthalmology

18 October 2010

Number of times this article has been viewed

Pia Allegri ${ }^{1}$

Antonio Mastromarino'

Piergiorgio Neri

'Uveitis Center, Ophthalmological Department of Lavagna Hospital, Genova, Italy; ${ }^{2}$ Uveitis Unit, The Eye Clinic, Azienda OspedalieroUniversitaria, Ospedali Riuniti di Ancona, Ancona, Italy
Correspondence: Pia Allegri

Uveitis Center Head, Ophthalmological

Department of Lavagna Hospital,

Corso Valparaiso 96/3, Chiavari 16043,

Genova, Italy

Tel +390I85329626

$\mathrm{Fax}+390185329627$

Email pallegri@asi4.liguria.it

\begin{abstract}
Curcumin has been successfully applied to treat inflammatory conditions in experimental research and in clinical trials. The purpose of our study is to evaluate the efficacy of an adjunctive-to-traditional treatment with Norflo tablets (curcumin-phosphatidylcholine complex; Meriva) administered twice a day in recurrent anterior uveitis of different etiologies. The study group consisted of 106 patients who completed a 12-month follow-up therapeutic period. We divided the patients into three main groups of different uveitis origin: group 1 (autoimmune uveitis), group 2 (herpetic uveitis), and group 3 (different etiologies of uveitis). The primary end point of our work was the evaluation of relapse frequency in all treated patients, before and after Norflo treatment, followed by the number of relapses in the three etiological groups. Wilcoxon signed-rank test showed a $P<0.001$ in all groups. The secondary end points were the evaluation of relapse severity and of the overall quality of life. The results showed that Norflo was well tolerated and could reduce eye discomfort symptoms and signs after a few weeks of treatment in more than $80 \%$ of patients. In conclusion, our study is the first to report the potential therapeutic role of curcumin and its efficacy in eye relapsing diseases, such as anterior uveitis, and points out other promising curcumin-related benefits in eye inflammatory and degenerative conditions, such as dry eye, maculopathy, glaucoma, and diabetic retinopathy.
\end{abstract}

Keywords: curcumin, anterior recurrent uveitis, phosphatidylcholine-bound-curcumin (Meriva)

\section{Introduction}

In recent clinical studies, curcumin showed its antioxidant, chemopreventive, and antiinflammatory activity in acute and chronic inflammation both in clinical practice (used in neurodegenerative, pulmonary, metabolic, autoimmune, and neoplastic diseases) and in experimental models. In the latter case, eg, Mohan et al showed that curcuminoids target fibroblast growth factor- 2 and inhibit expression of gelatinase $\mathrm{B}$ in the angiogenic process by using the cultured corneal cells of a transgenic mouse. ${ }^{9}$ In an experimental dry eye model, Chen et al investigated the anti-inflammatory effects of curcumin in curcumin pretreated cultured corneal epithelial cells exposed to hyperosmotic conditions. ${ }^{29}$ Kumar et $\mathrm{al}^{30}$ reported that curcumin feeding to rats with chemical-induced hyperglycemia can reduce oxidative stress that is the main cause of progression of cataract. ${ }^{1-12}$ The beneficial effects of this drug appear now to be related to the effect on upregulation of peroxisome proliferator-activated receptor- $\gamma(\operatorname{PPAR}-\gamma)$, which is a ligandinducible transcription factor involved mainly in controlling inflammation in peripheral organs. Activation of PPAR- $\gamma$ has been shown to control the response in microglial cells and limit inflammation. ${ }^{13-17}$ Curcumin has poor systemic availability, but recent studies have shown that the phosphatidylcholine formulation $\left(\right.$ Meriva $\left.^{\circledR}\right)$ increases its oral 
bioavailability. ${ }^{18-21}$ Yadav et al showed the inhibitory effect of curcumin on cell proliferation and production of cytokines, which are the main phenomena involved in inflammation. ${ }^{12}$ Many studies have been conducted on curcumin, but only Lal et al have shown the efficacy of curcumin on chronic anterior uveitis. ${ }^{22}$ Based on the previous data, we report our experience in patients suffering from relapsing anterior uveitis of different etiologies.

\section{Material and methods}

A total of 122 patients (68 men and 54 women) with recurrent anterior uveitis (RAU) were enrolled in the study. The age of the patients ranged from 21 to 68 (mean, $37 \pm 4.7$ ). All patients signed a written informed consent before entering into the study in accordance with the Helsinki International Treaty criteria. The patients included in the study were selected from those followed in our tertiary referral uveitis centre (Lavagna Hospital, Genova, Italy) in a previous period of about 2 years (mean \pm 3 months) and affected by RAU with $1-4$ relapses in a year. The RAU diagnosis was made by two different ophthalmologists with more experience on uveitis and was based on standard clinical criteria of International Uveitis Study Group (IUSG). ${ }^{23}$ Ophthalmological examination included each time: slit-lamp examination with IUSG grading of anterior segment cells and flare (from 0 to 4+), Early Treatment Diabetic Retinopathy Study (ETDRS) visual acuity measurement, intraocular pressure measurement, and fundus examination with Volk +90 lens. We developed a specific medical record in which, at each check-up visit, we registered the findings for each patient included in the study. Recurrent anterior bilateral or unilateral uveitis etiology was mainly of autoimmune origin (56 patients), followed by RAU of herpetic origin (28 patients), and of different or unknown origin (22 patients). All the patients received an orally administered product, Norflo ${ }^{\circledR}$ (Eye Pharma Co, Italy) containing $600 \mathrm{mg}$ of Meriva (Indena, Milano, Italy) in each tablet, at the dosage of two tablets/day during the follow-up period. An ongoing therapy with systemic drugs (steroids, immune-suppressants, antiherpetic, and antitoxoplasmic drugs) or eye drops (steroids, mydriatics, and cycloplegics nonsteroidal anti-inflammatory drug) was maintained, and Norflo tablets were added as an adjunctive treatment. The therapy was only administered to patients who had frequent relapses in the last 2 years of follow-up and was started at the time of a relapse. The follow-up period varied from 12 to 18 months (mean \pm 3 months), and 106 patients (61 men and 45 women) completed the study. All patients underwent complete ophthalmological examination at $0,7-15,30,90$,
180, and 360 days. The evaluation of the results at the end of the follow-up period was carried out by comparing the results before and after 1 year of follow-up in patients presenting $4,3,2$, or 1 relapses per year. We also studied the percentage of satisfaction and tolerance to additional therapy by means of a detailed questionnaire (included into the clinical record sheet) developed with the purpose of analyzing the patient tolerance and compliance to treatment at the end of 1 year Norflo therapy.

\section{Statistical analysis}

Frequency of total relapses, before and after 1 year of Norflo therapy, was assessed with Wilcoxon signed-rank test.

\section{Results}

We compared the results, before and after the treatment with Norflo, of the total number of patients with relapses and the number of total relapses.

As shown in Figure 1A, 106 patients (corresponding to the global number of patients included into the study) had relapses before the treatment with Norflo, and only 19 patients had relapses after the treatment with Norflo.

As shown in Figure 1B, there were in total 275 relapses 1 year before the treatment with Norflo and only 36 relapses at the end of the 12-month follow-up period, after the treatment with Norflo ( $88 \%$ of improvement).

Tables 1 and 2 show the detailed results before and after Norflo treatment in all treated patients, respectively. The table specifies the number of patients with 4, 3,2, or 1 relapses per year and the total number of relapses per year.

For instance, in Table 1 (before the treatment with Norflo) six patients had four relapses per year, which means a total number of 24 relapses per year. In Table 2 (after the treatment with Norflo) we can see that no patients had four relapses per year, with no relapses in 1 year. Therefore, the six patients who had four relapses per year before the treatment had zero relapses after Norflo additional therapy. In the second row of the same tables, 58 patients had three relapses per year, which means a total of 174 relapses before treatment, and only four patients had three relapses per year, which means a total of 12 relapses per year after treatment.

As shown in the tables, the results were highly significant with the Wilcoxon signed-rank test at a $P<0.001$ in all groups. There was also an improvement in symptoms and signs associated with relapses after Norflo treatment, including ocular pain, blurring of vision, pericorneal hyperemia and aqueous or vitreous cells, and flare in $42 \%$ of patients. Only seven patients worsened, and they required 
A

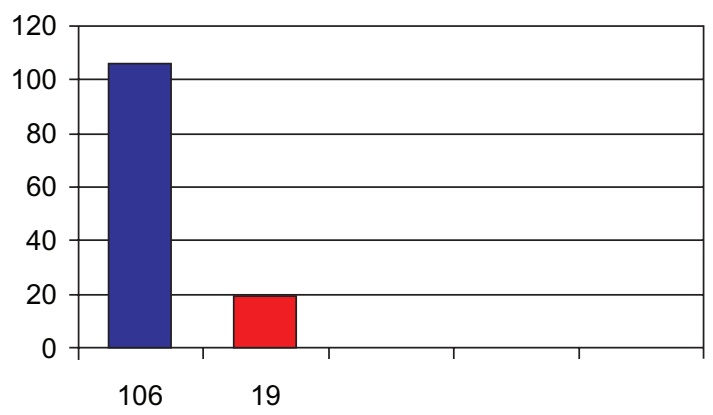

B

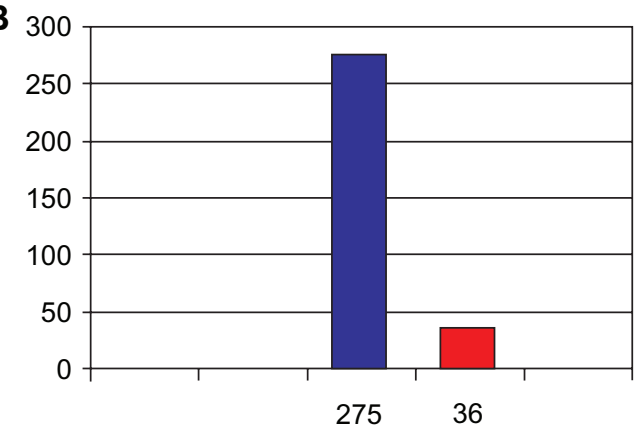

Figure I A) Total number of patients with relapses before and after Norflo therapy. B) Total relapse number before and after Norflo therapy. Note: Wilcoxon signed-rank test: $P<0.001$.

subtenon injections of steroids. Among the first 128 patients selected, only one subject had to interrupt therapy because of gastric intolerance related to reflux esophagitis. Fifteen patients dropped out of the study because of noncompliance with therapy or with follow-up visits. At the subjective symptoms and compliance questionnaire, $86 \%$ patients reported a subjective systemic well-being on evaluation progressively developed after 4-6 weeks of treatment and full compliance at the end of the follow-up period.

\section{Discussion}

In a recent review article Jacob et al studied curcumin's mechanism of anti-inflammatory activity both in vitro and in vivo preclinical studies. The author proved its beneficial anti-inflammatory and antivascular endothelial growth factor (VEGF) effects mediated by the upregulation of PPAR- $\gamma$ activation (curcumin is, in fact, a PPAR- $\gamma$ agonist). ${ }^{3}$ Immune cells mainly participating in ocular inflammation are the ones of glia and microglia. These cells, which participate in ocular inflammation, are controlled in their functions by PPAR- $\gamma$ action, which acts as a body metabolism sensor. ${ }^{13}$ Many ocular degenerative diseases that were not originally considered to be inflammatory (glaucoma, age-related macular

Table I Results obtained before Norflo treatment

\begin{tabular}{lll}
\hline $\begin{array}{l}\text { Number of } \\
\text { patients with } \\
\text { relapses }\end{array}$ & $\begin{array}{l}\text { Number of relapses } \\
\text { for each patient } \\
\text { per year }\end{array}$ & $\begin{array}{l}\text { Relapses per year } \\
\text { per group of } \\
\text { patients }\end{array}$ \\
\hline $6^{\mathrm{a}}$ & $4^{\mathrm{a}}$ & $24^{\mathrm{a}}$ \\
58 & 3 & 174 \\
35 & 2 & 70 \\
7 & 1 & 7 \\
$106^{\mathrm{b}}$ & - & $275^{\mathrm{b}}$ \\
\hline
\end{tabular}

Notes: ${ }^{a}$ Six patients enrolled four relapses each per year. This means that the total number of relapses for six patients was 24 in I year before Norflo treatment. b 06 patients enrolled had in I year a total of 275 relapses before Norflo treatment. degeneration, retinal ischemia, and diabetic retinopathy) are now considered from an inflammatory point of view. Therefore, the treatment of inflammation could represent an important target to manage ocular diseases. Based on these observations, conditions that are etiologically far from each other converge into the same inflammatory pathway. The beneficial effects of curcumin are suggested by epidemiological trials, supported by studies in animal models, and are extrapolated from in vivo studies but are not yet clinically validated, because the main disadvantages with curcumin therapy are its stability and bioavailability. Curcumin was recently complexated with phospholipids and the obtained product (Meriva) showed to improve at least 10 times the bioavailability vs uncomplexated curcumin in humans. ${ }^{19-21,24-26}$

After reviewing several studies on curcumin and on curcumin-phosphatidylcholine complex, some dealing on its anti-inflammatory effect in eye diseases, ${ }^{13-15,19,22,27-39}$ we decided to demonstrate Norflo efficacy as oral treatment of a typical chronic relapsing eye disease as RAU by means of a nonplacebo controlled study. It was an adjunct to standard treatment in 122 patients affected by RAU of different etiologies. All these patients completed at least 1 year followup, but 16 patients were excluded from the study; 15 of whom

Table 2 Results obtained after Norflo treatment

\begin{tabular}{lll}
\hline $\begin{array}{l}\text { Number of } \\
\text { patients with } \\
\text { relapses }\end{array}$ & $\begin{array}{l}\text { Number of relapses } \\
\text { for each patient } \\
\text { per year }\end{array}$ & $\begin{array}{l}\text { Relapses per year } \\
\text { per group of } \\
\text { patients }\end{array}$ \\
\hline 0 & 4 & 0 \\
$4^{\mathrm{a}}$ & $3^{\mathrm{a}}$ & $12^{\mathrm{a}}$ \\
9 & 2 & 18 \\
6 & 1 & 6 \\
$19^{\mathrm{b}}$ & - & $36^{\mathrm{b}}$ \\
\hline
\end{tabular}

Notes: aFour patients enrolled three relapses each per year. This means that the total number of relapses per year for four patients was 12 after Norflo treatment. ${ }^{b} \mid 9$ patients enrolled had in I year a total of 36 relapses after Norflo treatment. 
because of incomplete compliance with our therapeutic regimen or follow-up visits and one due to gastric intolerance related to reflux esophagitis syndrome. We included in our study only the patients followed for at least 2 years in our uveitis tertiary care out patient department who were affected by chronic relapsing anterior uveitis, which was difficult to manage because of high frequency and unpredictability of relapses. These subjects suffered from uveitis of different etiologies, among which we found three main different etiological groups: herpetic (varicella zoster virus, herpes simplex virus, cytomegalovirus, and Epstein-Barr virus) uveitis, autoimmune (sarcoidosis, overlap syndrome, systemic lupus erythematosus, and rheumatoid arthritis) inflammatory ocular disease, and various origin anterior uveitis ( 8 unknown origin, 7 toxoplasmic etiology, 3 tuberculosis, and 4 Lyme disease). Among these three groups, the most sensitive patients for treatment were autoimmune RAU, and the more relapsing patients were herpetic-derived RAU. This might be explained by the fact that herpetic uveitis is a mixture of autoimmune and viral origin diseases with typically relapsing syndromes. A continuous and protracted treatment gave the result of a good anti-inflammatory effect and prevention of relapses. In fact, in the first treatment period, we observed some relapses which disappeared in late, months of treatment. In our study, according to Malchiodi-Albedi et al, ${ }^{13}$ we suppose that the therapeutic efficacy of PPAR- $\gamma$ ligands is showed by the suppression and the improvement of clinical symptoms and decrease of clinical signs. From the point of view of side effects, we report only one patient who dropped out because of a gastric intolerance to curcumin tablets not reported in literature (we found only reports on curcumin-induced allergic contact dermatitis). ${ }^{31,32}$ The conclusion of our experience is that we can define curcumin as a bioactive, well-tolerated, and nontoxic therapy.

In the literature there is only one previous study on chronic anterior uveitis and curcumin treatment. It is a small study in which curcumin was orally administered at the daily dosage of $1.125 \mathrm{mg}$ to 36 patients who were divided into two groups: curcumin tablets and topical anti-inflammatory therapy and previous treatment plus anti-tuberculosis treatment. The results shown in this study were surprisingly favorable to curcumin-alone treated subjects. The researchers explained this important result by assuming that curcumin is as effective as the corticosteroid therapy, but without any evidence of side-effects. ${ }^{22}$

Our work showed for the first time that Meriva formulation permits us to reach active therapeutic levels in the eye at a common dosage of two tablets/day and is well tolerated.
Indeed, about $90 \%$ of the selected patients completed the 1 year follow-up. The success of our work suggests that curcumin's potential anti-inflammatory effect may be useful in other chronic or relapsing ocular surface diseases, such as dry eye syndrome, allergic conjunctivitis, and blepharitis. Chen et al investigated the anti-inflammatory efficacy of curcumin in treating in vitro dry eye syndrome model and concluded that curcumin has a potential therapeutic effect for treating this disease. ${ }^{29,30}$ Moreover, curcumin's therapeutic use in addition to traditional therapeutic protocols may be useful in retinal inflammatory diseases, such as macular edema or in retinal neovascular proliferative diseases, because curcumin is shown also to modulate angiogenesis. Uncontrolled angiogenesis had been associated with pathological eye conditions, such as diabetic retinopathy and choroidal or retinal neovascularization related to VEGF's effect. ${ }^{14,15,19,40-42}$ In fact, curcumin has been shown to prevent choroidal and retinal neovascularization in several experimental animal models, notably through the inhibition of VEGF receptor expression. ${ }^{15}$

Our trial has the main limit of a nonplacebo-controlled study, although we compared for statistical analysis a group of frequently recurring uveitis patients studied for 2 years before treatment and 1 year after treatment, who may be considered in the period before Norflo treatment as a control group, because we only add curcumin to previous standard therapy.

Another limit is the lack of standard measurements of anterior chamber inflammation by means of a laser cell flare meter, but the new instrument, actually available in commerce, has some structural defects which do not allow in all cases, a true value of intraocular inflammation in the case of small pupils because of synechiae and of opacities of the lens, which are typical of RAU.

\section{Conclusion}

Turmeric has been used for centuries to treat a lot of diseases including inflammatory diseases. The supplementation with curcumin, its active ingredient, has been shown to be safe in humans. The mechanism by which curcumin induces its anti-inflammatory effects is yet to be fully elucidated, but many studies have shown its relevance as a potent antiinflammatory and immuno-modulating agent. PPAR- $\gamma$ agonists (like curcumin) act on microglia and immune system cells modulating both innate and adaptive immune responses, and can have a profound effect on the inflammatory cascade. The positive results of our study show that Norflo (curcuminphosphatidylcholine complex) can play an important role in the adjunctive therapy of RAU of various origins and 
gives a contribution to the clinical potential efficacy of this plant-derived product in medicine.

\section{Acknowledgments}

The authors acknowledge Prof Ravera G of Statistical Analysis University of Genova, Dr Cagnola Aldo of Eye Pharma and Ms Capelli Maura our interpreter and translator, for their contribution to the study.

\section{Disclosure}

The authors report no conflicts of interest in this work.

\section{References}

1. Bengmark S. Curcumin an atoxic antioxidant and natural NFkappab, cycclooxygenase-2, lipooxygenase and inducible nitric oxide synthase inhibitor: a shield against acute and chronic diseases. J Par Ent Nutr. 2006;30(1):61-62.

2. Bright JJ. Curcumin and autoimmune disease. Adv Exp Med Biol. 2007;595:425-451.

3. Jacob A, Rongqian W, Zhou M, Wang P. Mechanism of the antiinflammatory effect of curcumin: PPAR- $\gamma$ activation. PPAR Res. 2007:89369.

4. Nonn L, Duong D, Preehl DM. Chemopreventive anti-inflammatory activities of curcumin and other phytochemicals mediated by MAP kinase phosphatase-5 in prostate cells. Carcinogenesis. 2007;28: 1188-1196.

5. Shakibaei M, John T, Schulze-Tanzil G, Lehmann I, Mobasheri A. Suppression of NF-kappaB activation by curcumin leads to inhibition of expression of cyclo-oxygenase- 2 and matrix-metalloproteinase- 9 in human articular chondrocytes: implications for the treatment of osteoarthritis. Biochem Pharmacol. 2007;73:1434-1445.

6. Strimpakos AS, Sharma RA. Curcumin: preventive and therapeutic properties in laboratory studies and clinical trials. Antioxid Redox Signal. 2008;10:511-545.

7. Holt PR, Katz S, Kirshoff R. Curcumin therapy in inflammatory bowel disease: a pilot study. Dig Dis Sci. 2005;50:2191-2193.

8. Jobin C, Bradham CA, Russo MP, et al. Curcumin blocks cytokinemediated NF-kappaB Activation pro-inflammatory gene expression by inhibiting inhibitory factor I-kappaB kinase activity. J Immunol. 1999;15:3474-3483.

9. Mohan R, Sivak J, Ashton P, et al. Curcuminoids inhibit the angiogenic response stimulated by fibroblast growth-factor-2, including expression of matrix metalloproteinase gelatinase B. J Biolog Chem. 2000;275(14):10405-10412.

10. Goel A, Kunnumakkara AB, Aggarwal BB. Curcumin as "curecumin": from kitchen to clinic. Biochem Pharmacol. 2008;75(4):787-809.

11. Biswas S, Rahman I. Modulation of steroid activity in chronic inflammation: a novel anti-inflammatory role for curcumin. Mol Nutr Food Res. Epub 2008 Mar 10.

12. Yadav VS, Mishra KP, Singh DP, Mehrotra S, Singh VK. Immunomodulatory effects of curcumin. Immunopharmacol Immunotoxicol. 2005;27(3):485-497.

13. Malchiodi-Albedi F, Matteucci A, Bernardo A, Minghetti L. PPAR- $\gamma$, microglial cells, and ocular inflammation: new venues for potential therapeutic approaches. PPAR res. 2008:295784.

14. Murata T, He S, Hangai M, et al. Peroxisome proliferator-activated receptor-gamma ligands inhibit choroidal neovascularization. Invest Ophthalmol Vis Sci. 2000;41(8):2309-2317.

15. Bonne C. PPAR gamma: a novel pharmacological target against retinal and choroidal neovascularization. $J$ Fr Ophtalmol. 2005;28(3):326-330.
16. Siddiqui $\mathrm{AM}$, Cui $\mathrm{X}, \mathrm{Wu} \mathrm{R}$, et al. The anti-inflammatory effect of curcumin in an experimental model of sepsis is mediated by upregulation of peroxisome proliferator-activated receptor- $\gamma$. Critical Care Med. 2006;34(7):1874-1882.

17. Jiang C, Ting T, Seed B. PPAR- $\gamma$ agonists inhibit production of monocyte inflammatory cytokines. Nature. 1998;391:82-86.

18. Cuomo J, Chernyshev V. Preliminary report-comparative bioavailability of curcuminoids from Meriva ${ }^{\circledR}$ vs $95 \%$ curcumin extract. Salt Lake City, UT: Usana Health Sciences, Inc; 2009.

19. Wan YJ, Pan MH, Cheng AL, et al. Stability of curcumin in buffer solutions and characterization of its degradation products. $J$ Pharm Biomed Anal. 1997;15(12):1867-1876.

20. Anand P, Kunnumakkara AB, Newman RA, Aggarwal BB. Bioavailability of curcumin: problems and promises. Mol Pharm. 2007; 4(6):807-818.

21. Marczylo TH, Verschoyle RD, Cooke DN, Morazzoni P, Steward WP, Gescher AJ. Comparison of systemic availability of curcumin with that of curcumin formulated with phosphatidylcholine. Cancer Chemoter Pharmacol. 2007;60:171-177.

22. Lal B, Kapoor AK, Asthana OP, et al. Efficacy of curcumin in the management of chronic anterior uveitis. Phytother Res. 1999; $13: 318-322$

23. Bloch-Michel E, Nussenblatt RB; International Uveitis Study Group Recommendations for the evaluation of intraocular inflammatory diseases. Am J Ophthalmol. 1987;103:234-235.

24. Li L, Braiteh FS, Kurzrock R. Liposome-encapsulated curcumin. In vitro and in vivo effects on proliferation, apoptosis, signaling and angiogenesis. Cancer. 2005;104(6);1322-1331.

25. Jurenka J. Anti-inflammatory properties of curcumin, a major constituent of Curcuma longa: a review of preclinical and clinical research. Alt Med Rev. 2009;14(2):141-153.

26. Marczylo TH, Steward WP, Gesher AJ. Rapid analysis of curcumin and curcumin metabolites in rat biomatrices using a novel ultraperformance liquid chromatography method. J Agric Food Chem. 2009;57(3):797-803.

27. Lal B, Kapoor K, Agrawal PK, Asthana OP, Srimal RC. Role of curcumin in idiopathic inflammatory orbital pseudotumours. Phytother Res. 2000;14(6):443-447.

28. Passos E, Grinstead RL, Khoobehi B. Effectiveness of curcumin, an angiogenesis inhibitor, in experimental choroidal neovascularization in rats. Invest Ophthalmol Vis Sci. 2002;43:E-Abstract 1274.

29. Chen M, Hu D-N, Pan Z, Lu CW, Xue CY, Aass I. Curcumin protects against hyperosmoticity-induced IL-1 $\beta$ elevation in human corneal epithelial cell via MAPK pathways. Exp Eye Res. 2010;90(3):437-443.

30. Kumar PA, Suryanarayana P, Reddy PY, Reddy GB. Modulation of $\alpha$-crystallin chaperone activity in diabetic rat lens by curcumin. Mol Vision. 2005;11:561-568.

31. Hata M, Sasaki E, Ota M, et al. Allergic contact dermatitis from curcumin (turmeric). Contact Dermatol. 1997;36:107-108.

32. Swierczynska MK, Krecisz B. Occupational skin changes in persons working in contact with food spices. Med Pr. 1998;49:187-190.

33. Sharma RA, Steward WP, Gesher AJ. Pharmacokinetics and pharmacodynamics of curcumin. Adv Exp Med Biol. 2007;595:453-470.

34. Lao CD, Ruffin MT, Normolle D, et al. Dose escalation of a curcuminoid formulation. BMC Complement Altern Med. 2006;6:10.

35. Bisht S, Feldmann G, Soni S, et al. Polymeric nanoparticle-encapsulated curcumin: a novel strategy for human cancer therapy. JNanobiotechnology. 2007;5:3.

36. Maiti K, Mukherjee K, Gantait A, Saha BP, Mukherjee PK. Curcuminphospholipid complex: preparation, therapeutic evaluation and pharmacokinetic study in rats. Int J Pharm. 2007;330:155-163.

37. Aggarwal BB, Sung B. Pharmacological basis for the role of curcumin in chronic diseases: an age-old spice with modern targets. Trends Pharmacol Sci. 2009;30(2):85-94.

38. Barry J, Fritz M, Brender JR. Determining the effects of lipophillic drugs on membrane structure by solid-state NMR spectroscopy-The case of the antioxidant curcumin. JAm Chem Soc. 2009;131(12):4490-4498. 
39. Aggarwal BB, Harikumar KB. Potential therapeutic effects of curcumin, the anti-inflammatory agent against neurodegenerative, cardiovascular, pulmonary, metabolic, autoimmune and neoplastic diseases. Int J Biochem Cell Biol. 2009;41(1):40-59.

40. Jeong SJ, Koh W, Lee EO, et al. Antiangiogenic phytochemicals and medical herbs. Phytotherapy Res. 2010 Jun 17 [Epub ahead of print].
41. Arbiser JL, Klauber N, Rohan R, et al. Curcumin is an in vivo inhibitor of angiogenesis. Mol Med. 1998;4(6);376-383.

42. Kowluru RA, Kanwar M. Effect of curcumin on retinal oxidative stress and inflammation in diabetes. Nutr Metab. 2007;4:8.
Clinical Ophthalmology

\section{Publish your work in this journal}

Clinical Ophthalmology is an international, peer-reviewed journal covering all subspecialties within ophthalmology. Key topics include: Optometry; Visual science; Pharmacology and drug therapy in eye diseases; Basic Sciences; Primary and Secondary eye care; Patient Safety and Quality of Care Improvements. This journal is indexed on

\section{Dovepress}

PubMed Central and CAS, and is the official journal of The Society of Clinical Ophthalmology (SCO). The manuscript management system is completely online and includes a very quick and fair peer-review system, which is all easy to use. Visit http://www.dovepress.com/ testimonials.php to read real quotes from published authors. 